\title{
Procesos reflexivos, desde las artes, sobre problemáticas de género en una escuela primaria de Santiago, Chile
}

Reflective processes, from the arts, about gender issues at a Primary School in Santiago, Chile

\section{Volumen 21, Número 2 \\ Mayo - Agosto \\ pp. 1-27}

Catalina Montenegro-González

Natalya Fierro-Navarro

Natalia Tardón-Gangas

\section{Citar este documento según modelo APA}

Montenegro-González, Catalina., Fierro-Navarro, Natalya., y Tardón-Gangas, Natalia. (2021).

Procesos reflexivos, desde las artes, sobre problemáticas de género en una escuela primaria de Santiago, Chile. Revista Actualidades Investigativas en Educación, 21(2), 127. Doi. 10.15517/aie.v21i2.46784 


\title{
Procesos reflexivos, desde las artes, sobre problemáticas de género en una escuela primaria de Santiago, Chile
}

Reflective processes, from the arts, about gender issues at a Primary School in Santiago, Chile

\author{
Catalina Montenegro-González \\ Natalya Fierro-Navarro² \\ Natalia Tardón-Gangas ${ }^{3}$
}

\begin{abstract}
Resumen: El presente artículo visibiliza el proceso de investigación e intervención en una escuela de la comuna de Santiago de Chile, a partir de dos unidades didácticas de Artes Visuales en sexto grado, donde se incorporaron problemáticas de género, como violencia y estereotipos. El objetivo de la investigación fue rediseñar, contextualizar y realizar intervenciones escolares en la asignatura de Artes Visuales, vinculadas a problemáticas de género presentes en los espacios cotidianos del estudiantado. La metodología de la investigación desarrollada durante 2018 y 2019 es una investigación-acción participativa. Los datos fueron recabados a través de la propia práctica docente y los instrumentos de evaluación dentro del aula (diagnósticos, círculos de conversación, trabajo artísticocreativo y presentación). La propuesta didáctica investigativa nos muestra la importancia de la integración de la reflexión en la creación artística, al lograr el objetivo propuesto. De esta manera, se abrió el espacio de debate sobre situaciones de violencia, la necesidad de valorar las experiencias personales vinculadas a temáticas de género, el respeto por la diversidad y la necesidad de construir aulas más inclusivas y respetuosas de la diversidad sexual y del género. Lo anterior da como principales resultados el desarrollo de una actividad artístico-reflexiva, el cuestionamiento de los estereotipos y las dinámicas sociales, además de propiciar el trabajo artístico-colaborativo como espacio de discusión, confianza y creatividad, dando relevancia al proceso por sobre los resultados, aspectos que perduraron en el grupo de estudiantes más allá de la asignatura de Artes Visuales.
\end{abstract}

Palabras clave: educación artística, procesos creativos, perspectiva de género, educación primaria.

Abstract: This article highlights the processes of research and intervention at a school located in Santiago of Chile, from two didactic units of Visual Arts in the sixth grade of Primary School, where gender trouble such as violence and stereotypes were incorporated. The purpose of this research was to redesign, contextualize and perform educational interventions in the Visual Arts subject, related to the gender issues that occur in the students' everyday places. The investigative methodology developed between 2018 and 2019, is a participatory action-research. All the data was collected through the teaching practice and evaluation instruments inside the classroom (diagnosis, focus group, artistic and creative work, and their presentation). The didactic research proposal shows us the importance of the integration of reflection in artistic creation, achieving the initial goal. Therefore, a space was opened for debate on situations of violence, the need to value personal experiences related to gender issues and the need to promote the value of the gender perspective, the respect for diversity and the need to build more classrooms that are inclusive and respectful of sexual and gender diversity. As main results the above gives us the development of an artisticreflective activity, questioning stereotypes and the social dynamics, in addition to promoting artistic-collaborative work as a space for discussion, confidence and creativity, giving relevance to the process over the results, aspects that persist in the group of students beyond the Visual Arts subject.

Keywords: art education, creative processes, gender perspective, primary school.

1 Universidad de Los Lagos, Osorno, Chile. Dirección electrónica: catalina.montenegro@ulagos.cl, ORCID https://orcid.org/0000-0002-4432-5582

2 Universidad Católica Silva Henríquez y Universidad Diego Portales, ambas en Santiago, Chile. Dirección electrónica: natalya.fierro@mail.udp.cl, ORCID https://orcid.org/0000-0001-9947-1686

${ }^{3}$ Universidad de O'Higgins y Universidad Católica Silva Henríquez, ambas en Santiago, Chile. Dirección electrónica: natalia.tardon@uoh.cl, ORCID https://orcid.org/0000-0002-9058-5916

Artículo recibido: 18 de noviembre, 2020

Enviado a corrección: 24 de febrero, 2021

Aprobado: 22 de marzo, 2021

Los contenidos de este artículo están bajo una licencia Creative Commons 


\section{Introducción}

El siguiente artículo muestra los resultados de un proyecto mancomunado entre una escuela primaria de Santiago de Chile y la Escuela de Educación Artística de una Universidad privada, las cuales, en conjunto, crearon el Proyecto Vincula, que tiene por objetivo principal rediseñar, contextualizar y realizar intervenciones escolares en la asignatura de Artes Visuales, con propuestas didácticas que surgen a partir de las siguientes preguntas ¿Cómo podemos rediseñar y contextualizar las actividades de la asignatura de Artes Visuales para que sea significativa al estudiantado? y ¿Cómo se incorpora la docente en el trabajo investigativo colaborativo? En el caso de este artículo se evidencia un proceso reflexivo, a través de los procesos creativos, sobre problemáticas de género presentes en los espacios cotidianos del estudiantado, asimismo una indagación sobre las posibilidades transformativas de estas actividades en la formación artística escolar, en una escuela primaria de Santiago de Chile.

El cruce entre educación y género en el contexto chileno- latinoamericano es un planteamiento que se da a partir del testimonio y relato de las experiencias didácticas implementadas en una escuela donde se llevaron a cabo procesos creativos en el marco de la asignatura de Artes Visuales durante los años 2018 y 2019. Dicho lo anterior, es importante comenzar este artículo hablando del cruce entre educación y género en un contexto latinoamericano-chileno, para continuar con las experiencias didácticas implementadas en una escuela en el marco de la asignatura de Artes Visuales.

Los estudios de género han tenido dentro de sus múltiples objetivos, visibilizar la marginación de la mujer desde todas las esferas sociales, además de las minorías sexuales y de género. Mimi Orner (1999), feminista postestructuralista, plantea la deconstrucción de los binarios, al ser estos los responsables de la subordinación de un concepto sobre otro, tal es el caso del binario hombre/mujer, el cual entrega el rol de subalterno a lo femenino, además de categorías preestablecidas que solo perpetúan un sistema reproductivo de desigualdades que reprime las características subjetivas. Como señala la autora, es necesario desde posturas postestructuralistas, examinar las posiciones binarias para recobrar posicionamientos invisibilizados y silenciados.

El cruce entre educación y género, entendido al último como una construcción social (Lamas, 1986), es lo que nos permite reconocer y visibilizar lo silenciado y borrado, la problematización de estos binarios y de lo no dicho, aquello que permite deconstruir, desde las artes, y resistir a las imposiciones de los estereotipos. Tal como lo plantea Mimi Orner 
(1999), es necesario producir este cuestionamiento, desnaturalizar lo que históricamente ha sido establecido como normal o natural y que responde a la narrativa de la otra persona.

Mimi Orner y sus estudios acerca de la escuela nos plantea la necesidad de abandonar las nociones esencialistas y reguladoras para comprender al estudiantado como sujetos. Según lo anterior, las propuestas didácticas que se evidencian en este artículo se aproximan a los intereses e inquietudes del estudiantado a partir de sus gustos musicales. Se consideró el contexto y se plantearon reflexiones del binario hombre/mujer que normalizan situaciones o conductas de violencia ¿Qué sucede cuando se permite cuestionar estas imposiciones en la sociedad desde la escuela? ¿Qué incidencia en los aprendizajes del estudiantado tiene incorporar elementos de género?

Según lo anterior, se propone una reflexión desde los posicionamientos de las pedagogías feministas y la coeducación, con una mirada territorial latinoamericana que permite articular el discurso desde la decolonialidad, entrecruzada con los procesos creativos, tomando consciencia de los espacios que habitamos y las personas con las que convivimos.

\section{Referentes teóricos}

Los recientes acontecimientos referidos a movimientos sociales y de protesta en Chile, como el denominado Mayo Feminista de 2018 (Liviana, 2020; Reyes-Housholder y Roque, 2019; Richard, 2018; Zerán, 2018) y el Estallido social de 2019 (Diaz-Romero, 2020; Gerber, 2020; Navarro, 2020) nos hacen revisitar y cuestionar no solo nuestras prácticas sociales, sino también las formas en que hacemos comunidad, nos relacionados o aprendemos. Es en este panorama donde se revelan prácticas, experiencias y saberes desde los feminismos en el continente americano, en el cual toman importancia aspectos como la decolonialidad, comprendida en palabras de Walsh como "un camino de lucha continuo en el cual se puede identificar, visibilizar y alentar "lugares" de exterioridad y construcciones alter-(n)ativas" (2014, p. 7), valorando la diferencia o lo marginado, y vinculando un territorio común, con una historia de movilización social en desarrollo. Lo anterior nos lleva a pensar en cuáles son las posibilidades reflexivas, creativas y de construcción del conocimiento en los espacios de aprendizaje, desde las miradas feministas en nuestro continente, y que en Chile tienen cada vez mayor notoriedad.

Con estas inquietudes, es posible abordar dimensiones colaborativas de los procesos de aprendizaje escolar, y concebir las prácticas artísticas colectivas dentro del aula como un espacio creativo, que da énfasis a la desjerarquización del aprendizaje, tal como señala Walsh 
(2014) a partir de la escucha de Mamá Dulu, procesos pedagógicos arraigados en las comunidades y la concepción de una "praxis político-pedagógica aferrada en la posibilidad y esperanza, y encaminada hacia la justicia, dignidad, libertad y humanización" (Mamá Dalu en Walsh, 2014, p. 11). Por su parte, Munévar (2015) se refiere a la necesidad de abrir los campos del saber en los procesos formativos universitarios en lo que define como la descolonización epistémica, en tanto se permite un tejido colectivo entre quienes conforman los espacios de aprendizaje para discutir sobre elementos de la inclusión de la diferencia o la consideración de las experiencias compartidas.

Este último concepto nos permite problematizar las prácticas docentes que nos afectan en todas las dimensiones de la vida y vivenciar las experiencias que constituyen el espacio de aprendizaje, este último como un lugar que va más allá del aula. Es decir, una pedagogía que se expande y se desborda desde los límites de la institucionalidad. Aquí toma relevancia el aprendizaje situado que Elliot Eisner define en relación con cómo las personas se sitúan "en un contexto social y material y este contexto, entendido como cultura, enseña" (2017, p. 125).

\subsection{Las pedagogías con perspectiva de género para los abordajes en el sur: caminando entre las pedagogías feministas y la coeducación}

Las propuestas de Bejarano, Martínez y Blanco (2019) se refieren a un camino donde se cruza el "feminismo y la educación" (p. 38), como consecuencia de las propuestas coeducativas, vinculadas a la igualdad, y una forma coherente de situar el pensamiento feminista pedagógico (Belausteguigoitia y Mingo, 1999; Cabello 2018; Maceira, 2008; Marí, et al., 2019; Sánchez, et al., 2016). Para una concepción de la educación que avanza hacia la coeducación, es necesario considerar los elementos que indican Subirats y Tomé (2013):

Modificar, al mismo tiempo, el modelo cultural y educativo, rebajando su androcentrismo [...] e incorporando elementos de la cultura de las mujeres, especialmente aquellos que tienden a disminuir el valor de la violencia y a fomentar las relaciones entre las personas y la conservación y el cuidado de la vida. (p. 70)

En el caso del presente artículo, que aborda una experiencia de investigación, tanto la investigadora como la docente a cargo del curso se posicionan desde la práctica de las pedagogías feministas en un contexto de aula mixta, lo que las lleva a reflexionar sobre el rol del feminismo en la formación del estudiantado heterogéneo. 
Bejarano et al. (2019), desde los posicionamientos pedagógicos feministas, plantean la relación entre el feminismo y la educación como un vínculo que se gesta "a partir de propuestas coeducativas asociadas al principio de igualdad de oportunidades" (p. 38). En este sentido, las autoras proponen posicionarse desde las reflexiones feministas, donde encuentra cabida la problematización del espacio escolar y sus relaciones de poder, en tanto reflejo de la sociedad hegemónica masculinizada y patriarcal, "que reproduce situaciones de desigualdad que alimentan estereotipos sociales" (Montenegro y Corvalán, 2020, p. 12). Así también las relaciones humanas sexistas y discriminatorias en el espacio de aprendizaje, apelan a una equidad de las condiciones de aprendizaje de aula. Ya que las reflexiones feministas apuntan a la visibilización de las mujeres, las diversidades sexuales y de género en este proceso, es que dependerá del contexto la toma de decisiones en cuanto a qué y cómo abordar el proceso de aprendizaje. En este caso, se transita desde las pedagogías feministas hacia la coeducación.

Retomando los planteamientos de Bejarano et al., (2019), existe concordancia con la propuesta de Montenegro (2018a) al establecer que todo espacio educativo puede ser abordado desde posturas feministas, a fin de propiciar lo que define como énfasis según el contexto en el que se está. Según esto, en el espacio de aula mixta es preciso hacer un énfasis en los principios coeducativos que den pertinencia al aprendizaje artístico en la formación de todo el estudiantado. Según esto, y desde posicionamientos feministas latinoamericanos, se espera principalmente cuestionar las relaciones de poder y todo acto discriminatorio y de marginación que encuentre espacio en el territorio escolar y que impida un desarrollo equitativo de la infancia y adolescencia. Según lo anterior, las posibilidades de concebir una escuela desde planteamientos coeducativos y feministas son viables por cuanto el espacio escolar específico pueda dar luces de cuáles son las mayores preocupaciones o propuestas que se deben abordar. En el caso de esta investigación, como indica Montenegro (2018a), la concepción de la escuela como un lugar de reproducción social, de un orden patriarcal y hegemónico, nos permite avanzar hacia una constitución escolar que se articula desde posturas feministas y entra en diálogo con los cuestionamientos que emergen de los movimientos desde 2018 en adelante en Chile (Seoane y Severino, 2019; Troncoso et al., 2019). De la misma manera, Montenegro (2019a) propone algunos elementos que se desprenden desde las pedagogías feministas y articulan la práctica docente e investigativa de este artículo, en el que la consideración de las singularidades del espacio escolar, serán claves a la hora de proponer una docencia e investigación significativas para el centro educativo. 
Algunos anclajes importantes son los que podemos contextualizar a nuestra realidad educativa actual, como por ejemplo, considerar las propias experiencias, narrativas y vidas en contextos singulares para aprender, hacerlo en relación y poseer un sentido colaborativo de los procesos de aprendizaje, todo esto bajo dinámicas de cuidado. (Montenegro, 2019a, p. 21)

En el contexto nacional chileno, concebir la formación desde los planteamientos de las pedagogías feministas hacia la coeducación, permite problematizar los elementos de menoscabo y discriminación de manera transversal en el espacio educativo, esto quiere decir que aún cuando contemplemos acciones concretas de valoración de la diversidad o la incorporación de referentes femeninas por ejemplo, existen diversos niveles de cuestionamiento, que nos llevarán no sólo a la utilización de lo dicho anteriormente, sino a identificar relaciones y discursos de poder asimétrico (tanto en el lenguaje como en los recursos, los temas, la organización, etc.), violencia simbólica, relaciones discriminatorias entre pares docentes, entre muchos otros elementos que permanecen subterráneos al espacio escolar y, que no serán develados en tanto no exista una voluntad legítima de cambiar y mejorar el ámbito educativo.

\subsection{Las experiencias artísticas como mediadoras para la transformación social}

El territorio escolar como un lugar que podría posibilitar los procesos reflexivos y de problematización de las discusiones sociales, permite establecer debates que afectan al estudiantado de manera directa como la discriminación por género, la marginación o la desigualdad. Esto podría permitir a la escuela, abordar las problemáticas que permean desde la sociedad de diversas maneras, siendo una propuesta, los procesos creativos, las que en un contexto escolar potencian el desarrollo de procesos de reflexión y experiencia, comprendiendo esta última en palabras de Teresa de Lauretis (1984) como:

El proceso por el cual se construye la subjetividad para todos los seres sociales. A través de ese proceso uno se ubica o es ubicado en la realidad social y de ese modo percibe y comprende como subjetivas (referidas a y originadas en uno mismo) esas relaciones. (p. 159)

\footnotetext{
${ }^{4}$ Las negritas son parte de la cita.
} 
Los lenguajes artísticos dan paso a la construcción del conocimiento, desprendido de la expectativa de un posible resultado, centrándose en el proceso, las relaciones y lo creativo en sí mismo. Esto permite dar valor a la experiencia artística en el contexto escolar como un acto mediador entre la expresión artística y las discusiones sociales actuales, como señalan GarcíaHuidobro y Montenegro (2020) con un sentido político y una experiencia transformadora. Al respecto, Montenegro complementa proponiendo una forma de "pensar el aprendizaje como una experiencia de encuentro" (2018b, p. 414), como una manera de aprender a ser (Faure et al., 1973), que posibilita las relaciones de confianza, alejadas de estereotipos y cercanas a las problemáticas de los propios contextos tanto educativos como sociales.

El acto mediador transformativo de los procesos creativos en el contexto escolar, serán un nudo entre los procesos creativos, la sociedad y la cultura que habitamos, lo que en palabras de Moreno (2016) considerará la "creación artística como un elemento generador de pensamiento y como un espacio de libertad del individuo" (p. 35), además de una práctica social que refleja la cultura y posibilita "la emergencia de diversas prácticas que no se alejen del momento histórico en el que se producen" (Ramos, 2019, p. 80) y de esta manera producir y construir conocimiento desde la propia colectividad, estableciendo formas específicas de construir conocimiento artísticamente. En la misma línea, se releva el rol mediador del arte como un elemento de transformación y cambio social (Barbosa, 2002; Rodrigo y Collados, 2015), que da importancia al carácter colaborativo de las experiencias y valora las colectividades, estableciendo relaciones de confianza entre pares, permitiendo un desarrollo basado en la práctica dialógica (Rodríguez, 2015). Al respecto, Palacios (2009) plantea la necesidad de hacer propuestas considerando las prácticas artístico-culturales desde una perspectiva comunitaria, donde se dé énfasis a la concepción del arte para el cambio social y le reste protagonismo a la obra.

Es posible entonces, centrar las prácticas artísticas más cercanas al bienestar social y elementos del activismo político "y menos sujetos a consideraciones estéticas o críticas" (Palacios, 2009, p. 203). Según lo anterior, es posible identificar algunos elementos que transitan desde y hacia las artes como espacios formativos y de la sociedad general como el respeto, la colaboración y la equidad (Huerta, 2016). Esto concuerda con las ideas del arte comunitario en sus inicios en las décadas de los sesenta y setenta en Inglaterra donde se enfatiza el proceso de aprendizaje colectivo (Birchall, 2017) lo que se plantea como "espacios de devenir o agenciamiento, en los que el trabajo es producido de forma transversal, rompiendo las jerarquías impuestas por la modernidad y abogando por una continua 
apropiación y dispersión de la cultura" (Blanco, 2001, p. 15). Según esto, es posible visualizar una investigación que concibe las prácticas artístico-culturales como agentes articuladores de subjetividades, vinculadas a problemáticas de género en la contemporaneidad nacional chilena.

\section{Metodología}

La metodología de la investigación desarrollada entre 2018 - 2019, está definida como una Investigación-Acción Participativa (Flores-Kastanis, Montoya-Vargas y Suárez, 2009; Lewin, 1946) vinculada a los procesos artístico-creativos de aula (Ledezma et al., 2017) estableciendo relaciones de colaboratividad entre investigadoras, docentes y estudiantes para llevar adelante el proyecto en una relación desjerarquizada.

\subsection{Enfoque}

El enfoque de la investigación se define como cualitativo. Para complementar lo anterior, es necesario especificar algunas de las características del contexto en el cual se desarrolla la investigación. El colegio primario de la región Metropolitana-Chile, posee altos índices de estudiantes migrantes (70\%), provenientes de variados sectores socioeconómicos. En este contexto, Vincula entrecruza tres figuras para el desarrollo de su proyecto, por una parte la docente de la asignatura de Artes Visuales, quien desarrolla docencia en sexto grado, la investigadora y las dos asistentes quienes son estudiantes de la Escuela de Educación Artística de la Universidad, alumnas de la investigadora y practicante de la profesora a cargo de la asignatura, todas conforman en equipo de investigación, quienes establecieron acciones conjuntas de diseño didáctico (Investigadora y docente), docencia (profesora a cargo de la asignatura de Artes Visuales) y recogida de datos (estudiantes en práctica). De esta manera, se llevan a cabo las acciones mediante el desarrollo de las actividades de las unidades para la asignatura de Artes Visuales propuestas por el Ministerio de Educación (MINEDUC). Se proponen herramientas desde una postura feminista de investigación, como indica Mohanty, metodologías analíticas feministas que permiten visibilizar las relaciones de poder y como un ejercicio que da un tratamiento más inclusivo "para una reflexión sobre la justicia social" (Mohanty, 2008, p. 420).

Esto cobra relevancia con las propuestas didácticas feministas para la enseñanza de las Artes Visuales (Montenegro, 2018a), desde donde se desprende la necesidad de articular una investigación que se hace parte del contexto y sus posibilidades de cambio a través del énfasis 
en la formación coeducativa (Bejarano et al., 2019; Subirats, 2017; Subirats y Tomé, 2013; Tomé y Rambla, 2001). Lo anterior, nos permite establecer dinámicas de diálogo crítico en el espacio formativo, "La reflexividad no busca la homogeneidad de experiencias, ni de pensamientos, por el contrario, persigue la multiplicidad de voces, el diálogo en distintos niveles para poder llevar a cabo una reflexión" (Montenegro, 2015, p. 49), lo que Macbeth (2001) propone como una "polisemia de múltiples posiciones, intereses y agencias" (p. 39).

\subsection{Unidades de análisis}

La aplicación de las unidades tuvo una extensión total de tiempo de siete meses, analizando un curso como muestra probabilística, conformado por 36 estudiantes de primaria (6to básico en Chile) con una edad promedio de 11 años. Se consideran estas unidades de análisis por dos razones: i) El contenido curricular de la asignatura de Artes Visuales que permitiera una articulación entre el enfoque de género y la disciplina y ii) el horario de clases que permitía la participación de todo el equipo de investigación con la docente a cargo de la asignatura.

Según lo anterior, se definen algunas acciones clave para el desarrollo del trabajo de campo con el grupo de estudiantes, i) Análisis de las propuestas curriculares del Ministerio de Educación chileno, para dar énfasis a los contenidos que cuestionen y problematicen los estereotipos de género y temáticas de violencia de género. Esto realizado a través del desarrollo de clases dialógicas y reflexivas diseñadas por la investigadora y la profesora de la escuela, ii) Consideración de las propias problemáticas, preocupaciones, imaginarios 0 prejuicios del estudiantado y iii) Desarrollo de actividades artísticas que potencien la relación respetuosa entre pares y que propicie la reflexión constante a partir del desarrollo artístico creativo. Este punto se considera desde los planteamientos de los Talleres de reflexión artístico visual (Montenegro, 2019b; 2019c), los que posibilitan un encuentro colectivo y cooperativo de reflexión en torno a las preocupaciones sociales, teniendo el proceso artístico como lenguaje. Esto persigue la desjerarquización de los procesos de aprendizaje, para construir colectivamente un tejido, materializado a través de una práctica artística colaborativa tanto en pequeños grupos (caso 1) como en la colectividad del curso (caso 2). A lo anterior, se suman algunos requerimientos evaluativos por parte de la escuela, lo que nos lleva a organizar las experiencias contemplando instrumentos de recogida de información que puedan servir para el proceso de evaluación. 
Al realizar la investigación con estudiantado menor de edad, se realizó un proceso de autorización por parte de padres madres y personas cuidadoras principales para tener el consentimiento informado tanto de ellos y ellas como del grupo de estudiantes. El proceso de recogida de datos y trabajo de aula con la unidad de análisis se realizó entre los meses de marzo y diciembre (ambos inclusive) de 2018. Para posteriormente realizar el análisis de los datos recogidos durante el segundo semestre de 2018 y 2019.

\subsection{Técnicas de recolección}

Las técnicas de recolección y construcción de los datos estuvieron definidas por: i) Diagnóstico escrito al inicio de las actividades para definir los conocimientos previos del curso respecto de las temáticas que se abordarían. En este caso se desarrolló individualmente a través de preguntas de desarrollo. ii) Creación de obras artísticas de carácter colaborativo con su respectiva evaluación donde en el caso 1 se proponen afiches y en el caso 2 bordados, iii) Círculos de conversación reflexivo al final de las actividades artísticas donde se retoman los temas trabajados en los diagnósticos. En ambos casos que serán expuesto más adelante, se realizaron las tres etapas descritas.

\subsection{Procedimiento de análisis}

El procedimiento de análisis se establece como una discusión analítica de los datos recogidos y producidos, teniendo un carácter de tipo cualitativo. Se establecieron categorías de análisis emergentes del diagnóstico inicial de ambos casos. En la segunda actividad se da mayor énfasis a la experiencia artística-creativa puesto que a partir del círculo de conversación reflexivo final de la primera actividad, se advierten dominios conceptuales y reflexivos que permitieron a las investigadoras dar espacio al proceso artístico-reflexivo (Montenegro, 2019b, 2019c).

En ambos casos se lleva a cabo un análisis triangulado de los datos desde la construcción de una matriz

\section{Resultados}

A continuación, se profundizará en las dos experiencias escolares que tienen como temas centrales problemáticas de género, para abordar la reflexión desde los procesos creativos. 


\subsection{Descripción de las experiencias}

Las experiencias que constituyen este artículo, se organizan a través de un eje articulador en ambas actividades: las problemáticas de género, evidenciando elementos como discriminación, desigualdad, marginación, estereotipos y violencia, además del cuestionamiento de conceptos como la masculinidad hegemónica comprendida como "la posición que ocupan los hombres en este orden de género" (Muñoz y Lira, 2020, p. 2). Lo anterior se propone a partir de situaciones discriminatorias vividas en la escuela y la necesidad de establecer procesos reflexivos significativos que permitieran la apropiación de los temas en el grupo de estudiantes.

\subsubsection{Caso 1, Violencia de género e imaginarios desde la música reggaetón}

La primera experiencia fue concebida como un desplazamiento de la Unidad propuesta por el Ministerio de Educación, siendo la Unidad 2: La escultura como medio de expresión y creación. Realización de trabajos artísticos que usen el volumen y el espacio (MINEDUC, 2013, p.73). Con esto se propone una actividad vinculada al Reggaetón, la cultura visual estudiantil y sus posibles relaciones con la violencia de género. A través de una conversación inicial, se decide con el curso realizar una serie de afiches volumétricos para llevar a cabo una campaña al interior de la escuela para condenar la violencia. Para llevar a cabo la actividad, el estudiantado hizo el siguiente ejercicio como activador del debate: durante algunos meses, el curso realizaba una lista con canciones que la profesora programaba con un parlante, canciones que escuchaban durante la clase de Artes Visuales, semana tras semana. Al revisar los títulos, la docente a cargo del curso, advirtió también el contenido violento de muchas de las canciones, y comenzó la lectura de las letras para proponer un trabajo crítico desde las líricas de las canciones. Es así como la profesora propone la proyección de las letras en pantalla gigante y su lectura sin música. Esto dio paso al cuestionamiento en una primera sesión de clases sobre los estereotipos de género y descripciones de violencia explícita y simbólica en los textos. La actividad provocó extrañeza en el grupo de estudiantes, el que se vio enfrentado a una relectura de su cotidianeidad, a partir de las canciones que escuchaban. 
Figura 1: Canciones sugeridas por el curso para su escucha durante las clases de Artes Visuales. Santiago, Chile, 2018

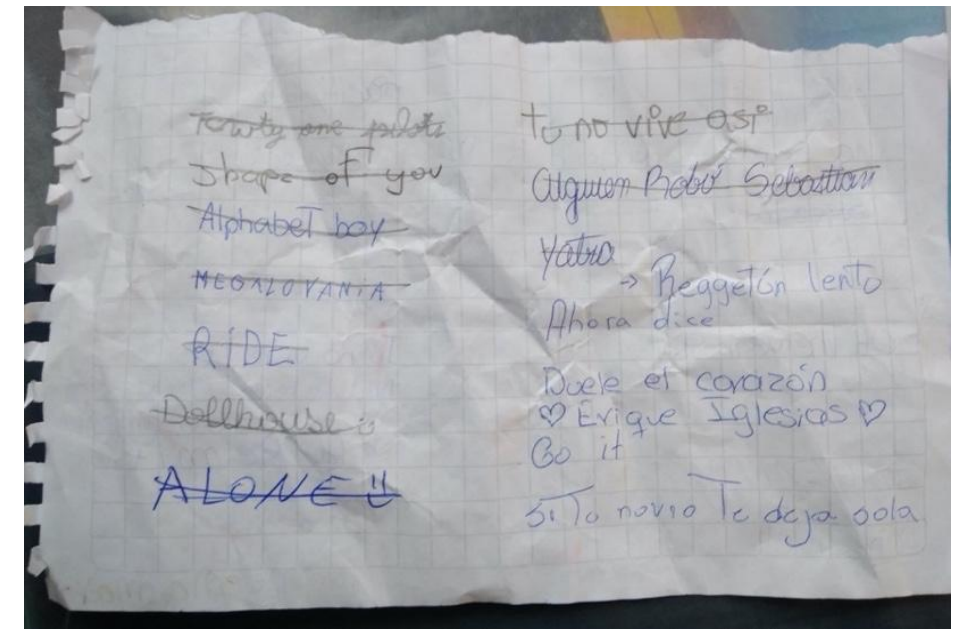

Fuente: Elaboración propia

Este ejercicio de lectura descontextualizada, permitió comenzar nuevas reflexiones para el estudiantado a partir de elementos cotidianos de su vida, como la música. Con esto se llevó a cabo un proceso diagnóstico, no solo de la actividad que se realizaría, sino también como una forma de situar las actividades que vendrían desde aspectos teóricos y del conocimiento que tenía el estudiantado respecto de las temáticas a tratar. Esta acción consistió en un cuestionario donde se pudo indagar sobre la relación del estudiantado con canciones de reggaetón. Se les propuso algunas preguntas en torno al reggaetón, el género y posibles situaciones vinculadas a la violencia de género que pudieran observar a través de las canciones que escuchaban, tanto en el espacio escolar como fuera de él.

A partir de esta actividad se pueden identificar los primeros cuestionamientos en relación a la música y sus letras, llevando a cabo un proceso reflexivo que problematiza sus propias prácticas adolescentes, las que Tenti (2000) define como construcciones sociales y, por tanto, será la sociedad también la que permita a las personas sentirse aceptadas en el grupo. A partir del diagnóstico, se organizaron las respuestas desde dos categorías: 1) Ritmo y 2) Letras.

\subsubsection{Categoría ritmo}

El estudiantado plantea una diferenciación entre el ritmo y las letras, teniendo algunas dificultades en las segundas, ya sea por el idioma (diferente al castellano) o porque no conocen las palabras que varían dentro de la lengua castellana según los países. Dentro de las respuestas más recurrentes del diagnóstico se encuentra el gusto por el ritmo de las canciones 
y lo pegajosas que pueden ser. Esto se puede observar en las frases que se repiten con mayor fuerza en el diagnóstico respecto de la pregunta ¿Te gustan las canciones de reggaetón? A esto responden:

Lo que me gusta del reggaetón es que tiene buen ritmo y lo que no me gusta es la letra (Estudiante 1)

Esta cita identifica a casi la totalidad del curso, exceptuando un pequeño grupo (4 a 5 estudiantes) que gustan más de la música K-Pop. Otra estudiante refuerza la idea del gusto por el ritmo cuando señala que:

No me gustan las letras, pero el ritmo sí (Estudiante 2)

Existe una concordancia entre el estudiantado respecto del valor que se hace al ritmo por sobre otros elementos como la letra de las canciones. Se evidencia que no existe una reflexión profunda para seleccionar las canciones, sino que existen otros motivos como; aceptación colectiva, disfrute personal del ritmo y otros. El desafío de la actividad es llevar esta reflexión al proceso de creación artística y se traslada la discusión a las posibilidades que puede tener en el campo visual.

\subsubsection{Categoría Letras}

Esta categoría despertó en primera instancia, los cuestionamientos por parte del estudiantado, bajo el argumento de una afinidad rítmica, pero no del contenido de las letras. Lo anterior, concuerda con la idea de una apropiación colectiva de las canciones, pero también como un detonante de reflexión crítica. De esta manera, separan y evidencian sus incomodidades frente a las líricas:

Lo que no me gusta del reggaetón es la letra. (Estudiante 9)

No me gustan las letras (Estudiante 19)

A partir de las textualidades iniciales de este diagnóstico, el equipo de docentes e investigadoras propone al curso la actividad titulada Afiches para la prevención, poniendo en el centro del proceso analítico-crítico elementos de las canciones vinculados a la violencia de género que el curso pudo identificar. Para responder a dos cuestiones clave que las docentes investigadoras identifican como relevantes: i) la necesidad de establecer canales no solo de reflexión, sino también de prevención de la violencia de género en todas sus formas y que en 
el caso de las canciones será de tipo simbólica, en lo que Bourdieu (2000) define como poder oculto o violencia simbólica, donde existe una asimetría y relaciones de poder que ejercen violencia no evidente o inconsciente, ii) La necesidad de responder a los requerimientos de la escuela, en cuanto a procesos artísticos evaluables y es por esto que la actividad se enmarca en la unidad 2 anteriormente descrita.

Al identificar esos aspectos se hace necesario llevar a cabo un proceso de identificación de estereotipos de género presentes no sólo en las canciones, sino también en la vida cotidiana. Se problematizó tanto sus propias experiencias como las de sus familias, amistades y docentes, llegando a discutir sobre profesiones, labores del hogar o incluso actitudes y formas de vestir.

Una vez que se organizan conceptualmente las discusiones, el estudiantado desarrolla sus propuestas visuales. Surge como idea del curso, tomar las letras de las canciones para desarrollar las propuestas visuales, dando énfasis a los elementos más relevantes del afiche a través del volumen.

Figura 2: Propuestas de afiches sobre canciones de reggaetón, elaborados por los-as estudiantes. Trabajo en proceso Santiago, Chile, 2018.
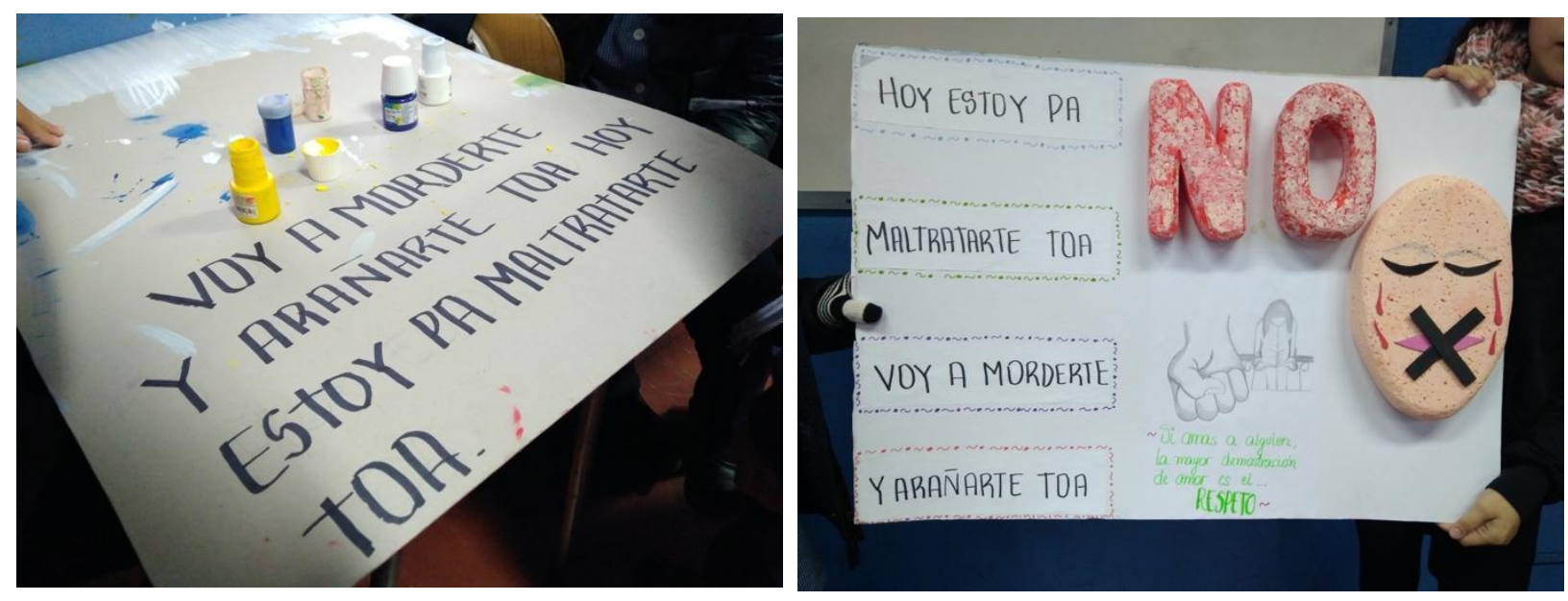

Fuente: Elaboración propia.

El trabajo desarrollado permitió que el estudiantado pudiera identificar los elementos de violencia implícita que transmiten las letras de las canciones. El diálogo se fue centrando en el cuestionamiento de lo establecido en distintos ámbitos de su cotidianidad que reprodujeran situaciones de desigualdad y violencia. Las alumnas fueron desarrollando un empoderamiento que les permitió cuestionar y dar argumentos sólidos respecto de la decisión del equipo 
directivo, al imponerles un vestuario en una ceremonia formal que obligaba al uso del jumper por ser mujer.

\subsubsection{Caso 2. Estereotipos y discriminación: Bordando los prejuicios}

El caso 2, es una actividad que se desarrolló durante el segundo semestre, posterior a la actividad del caso 1 con el mismo grupo de estudiantes. La Unidad 4, Entorno cultural: el hombre contemporáneo y la ciudad. Los objetivos trabajados fueron: 1. Crear trabajos de arte y diseños a partir de diferentes desafíos y temas del entorno cultural y artístico a partir de técnicas mixtas, 2. Evaluar críticamente trabajos de arte y diseño personales y de sus pares considerando: expresión de emociones y problemáticas sociales, uso de materiales y procedimientos, aplicación de elementos del lenguaje visual, propósitos expresivos.

Al igual que en el primer caso, se realizó un diagnóstico que apuntaba a conocer los niveles de conocimiento del estudiantado respecto de temáticas de género referidas a estereotipos, entendiendo estos como un conjunto de representaciones, opiniones o juicios respecto de ser hombre o mujer (la discusión no alcanzó a proyectarse a géneros no binarios o variables). En ese sentido, se buscaba indagar sobre los estereotipos a los que el estudiantado se sentía expuesto o respondía desde experiencias personales, familiares, tradiciones sociales, entre otras. El diagnóstico arrojó que más del 80\% del curso comprendía lo que significaba un estereotipo o un prejuicio, y se advierte una comprensión respecto del rol de estos conceptos en la sociedad y sus características nocivas al momento de situar la discusión en temáticas de género. Algunas de las ideas que emergen desde el diagnóstico son por ejemplo la definición de prejuicio como:

Cuando uno hace un juicio sin conocer (Estudiante 2); Es criticar a alguien antes de conocerlo (estudiante 9); Es hablar de alguien sin conocerla (Estudiante 32)

Mientras que, al preguntar por el concepto de estereotipo, las respuestas son menos precisas que con el concepto anterior, pero se acercan a posibilidades reflexivas:

Es como la idea de algo (Estudiante 10); Por ejemplo: tengo una imagen y muestra un modelo o una idea de algo (Estudiante 19); Algo copiado o algo como ejemplar (Estudiante 32).

Con esto, hubo una tercera definición que se centra en los estereotipos de género y es desde donde emerge la propuesta creativa. Aquí algunas ideas que se destacan son: 
Ejemplo: A las niñas les gusta las muñecas (Estudiante 3); Podría ser como la mujer ideal o el hombre ideal (estudiante 9); Los modelos, todas las mujeres quieren ser como ellas

(Estudiante 36).

Se aprecia un acercamiento espontáneo al concepto, que se verbaliza principalmente a través de ejemplos y muestran la relación que logran establecer entre los ejemplos, es decir, lo que comprenden por estereotipos, donde no se visibilizan identidades de género no binario o variable.

A partir de la discusión dada en la primera sesión donde también se vieron y cuestionaron ejemplos de la publicidad y la televisión, definieron como curso llevar a cabo una actividad que hiciera referencia a los estereotipos de género, no solo en su tema, sino también en su materialidad. De esta manera, realizaron bordados en tela como un acto reivindicativo de las labores estereotípicamente femeninas y como reflejo de las reflexiones pre-adolescentes respecto de las actividades impuestas socialmente según género. Lo anterior, configura una actividad que se organiza tomando dos elementos de la propuesta del Ministerio de las Culturas, las Artes y el Patrimonio (2020): Contenidos y Metodología, donde se sugiere en la primera "cuestionar el imaginario colectivo sobre el género para desarrollar un ejercicio deconstructivo sobre los significados sociales de género, presentes en los discursos y materias escolares" (Mincap, 2020, p. 21). Por otra parte, en la dimensión metodológica se sugiere "vincular los contenidos con experiencias personales y desarrollar formas de trabajo colaborativo" (Mincap, 2020, p. 24). Al considerar lo anterior, es posible advertir de manera constante el relato del estudiantado respecto a las cosas para niños y niñas y las actividades que es posible hacer cuestionando estas divisiones o roles. 
Figura 3: Ejemplos de bordado individual, elaborados por los-as estudiantes. Santiago, Chile, 2018
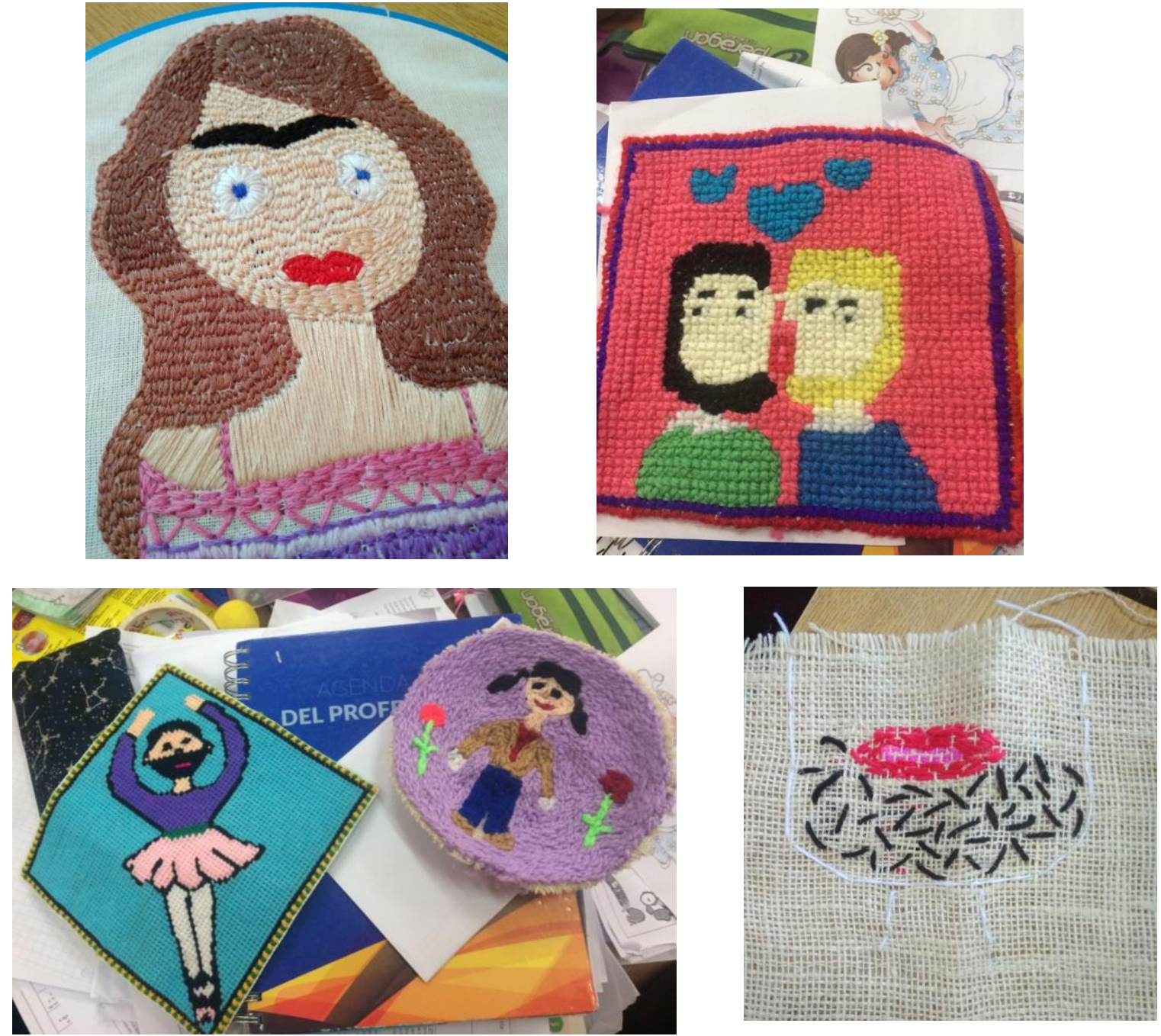

Fuente: Elaboración propia.

Al finalizar esta actividad, se develan reflexiones por parte del estudiantado que apuntan al cuestionamiento del deber ser social, a la libertad de expresión y a la necesidad de construir espacios escolares más libres y ausentes de estereotipos, que limitan sobre todo a las estudiantes mujeres. Al momento de presentar los bordados, se retoman las consideraciones que motivaron su realización, tanto a nivel material como temático y se volvieron a discutir los temas planteados y, cómo ellos y ellas, ahora desde lo aprendido, combatían estas imposiciones sociales. Se evidencia a través del trabajo artístico, la valoración por la diversidad y el respeto por la diferencia (Figura 3) plasmando en los bordados, imágenes tanto de cuestionamiento de estereotipos físicos, de oficios y de orientaciones sexuales. 
Figura 4: Proceso de montaje colectivo. Presentación final. Santiago, Chile, 2018

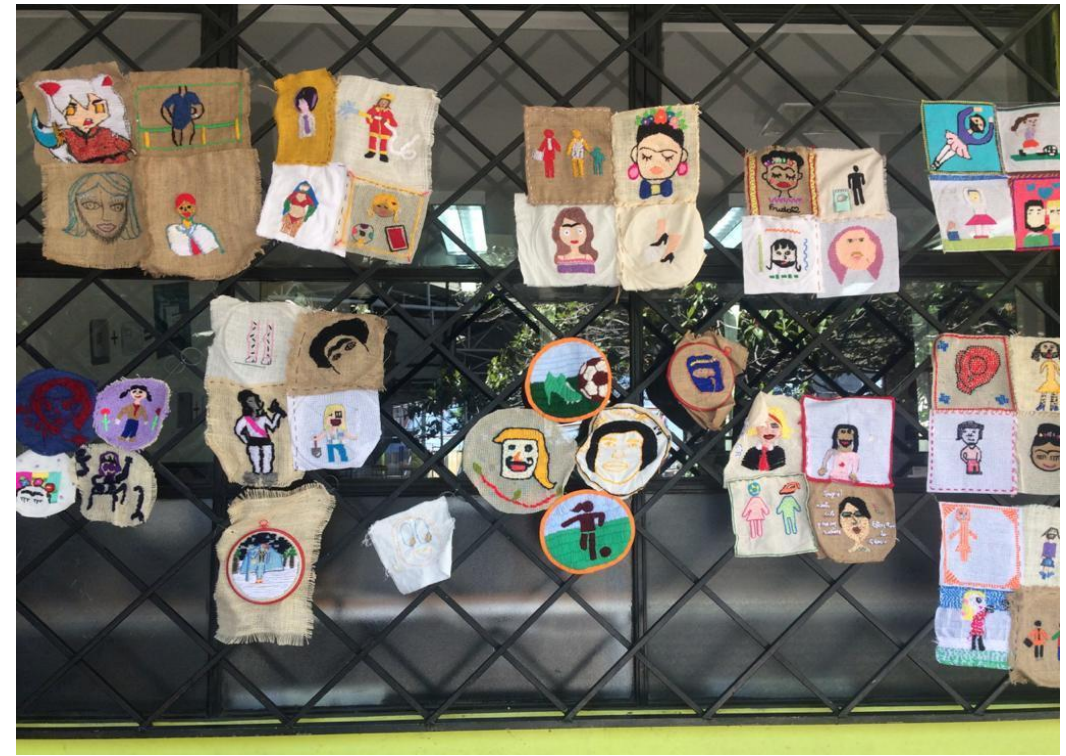

Fuente: Elaboración propia

Este proceso de aprendizaje nos permitió tanto como docente, investigadoras y estudiantes, cuestionar los roles dentro de la escuela y la sociedad. El trabajo y desarrollo de un ambiente basado en el respeto y la valoración de la diversidad que fomente el desarrollo de subjetividades libres de estereotipos, los preparó para en el 2019 ser un grupo que comprende lo subjetivo como algo esencial dentro de su desarrollo, esto les permitió acoger desde lo afectivo a un compañero que decide asumir un proceso de transformación y tránsito a lo no binario.

\subsection{Hallazgos, resultados y nuevos desafíos}

Las experiencias, que como se indicó anteriormente, se realizan a lo largo de un año, durante el primer y segundo semestre, articularon el trabajo a partir de propuestas reflexivas artístico visuales (Montenegro, 2019b, 2019c) donde se lleva a cabo una experiencia que permite dar cuenta de la relación entre las preocupaciones sociales y los procesos creativos para llevar a cabo esta reflexión, dando énfasis a los lenguajes artísticos para ir más allá de la oralidad o la escritura. Con lo anterior, se logró incorporar las experiencias y la multiplicidad de las personas que forman parte del espacio de aula, para reflexionar sobre sí mismas, pero también para cuestionar, lo que Munévar (2015) define como "la apreciación histórica monolítica, lineal, irreversible, única y unidireccional del pensamiento binario inscrito en las 
normas" (p. 70), lo que repercute en las formas de conocer el mundo y en las maneras que se cuestionan los procesos de enseñanza y aprendizaje. De esto se da cuenta en el proceso creativo artístico de ambas actividades, pero también en los círculos de conversación reflexiva donde emergieron categorías de análisis tan complejas como violencia de género, estereotipos y responsabilidad en su construcción pero también elementos que nos hacen pensar en las posibilidades de cambio social como la construcción de una escuela equitativa y la apropiación de actividades escolares que no tengan etiquetas de género.

El proceso de investigación permite indagar sobre las posibilidades que otorga el cuestionamiento de actividades, que ven en el cotidiano y que están presentes en todos los ámbitos de sus vidas. La reflexión artística permitió desarrollar una actividad reflexiva que se complementa con el proceso verbal y escritural a través de lenguajes artísticos y, que da espacio a desarrollar procesos expresivos, artísticos y reflexivos más allá de la oralidad o actividades de debate o plenario. Esto puede ser una oportunidad de incorporación del estudiantado que tiene mayor dificultad para integrarse en las discusiones del curso. Por otra parte, ambas actividades empujaron al grupo curso a realizar reflexiones donde cuestionaban su propio entorno, lo que les llevó a repensar sus dinámicas sociales y a pensar en la necesidad de desmarcarse tanto de situaciones de violencia de género como de responder a roles y estereotipos. Esto, si bien pudo abrir un espacio reflexivo, es difícil de identificar individualmente, sobre todo en las actividades de círculos de conversación reflexivo, puesto que cada idea planteada puede ser tomada también por el resto del estudiantado y queda la incertidumbre de si efectivamente es una reflexión que les hace eco personalmente o solo siguen el discurso del grupo. Esto podría ser un sesgo importante en la investigación e invita pensar nuestros instrumentos de recogida de datos que permitan evidenciar un cambio legítimo en las personas más allá de una declaración. Sin embargo, las actividades si bien tenían elementos de trabajo autónomo, propiciaron el trabajo artístico colaborativo como un espacio de discusión, confianza, pero también de creatividad, dando relevancia al proceso más allá de los resultados y que se presenta como señala Barbosa (2002) como elementos que empujan hacia la transformación y el cambio social. Asimismo, las actividades que conforman este artículo tuvieron repercusiones posteriores en el grupo de estudiantes, que se pudieron observar a través del relato de la maestra tutora del grupo y que apuntan a un curso más colaborativo, respetuoso y que dio continuidad a actividades de bordado en algunos varones del grupo. Según lo anterior, la actividad responde a lo que plantea Palacios (2009) 
cuando señala la necesidad de concebir las prácticas artístico-culturales dando énfasis al cambio social, buscando la importancia de un proceso significativo, más que resultados.

Es importante mencionar también, que las actividades estuvieron marcadas por las propias experiencias y los contextos que el estudiantado habita (Montenegro, 2018a), evidenciando reflexiones que parten desde un posicionamiento latinoamericano, esto último considerando que aproximadamente el 70\% del grupo curso es estudiantado migrante. Esto se vincula con lo que Munévar (2015) plantea como descolonización epistémica y permite dar paso a una actividad colectiva para abordar temáticas sobre los estereotipos, los roles y violencia de género y la necesidad de dialogar en torno a dinámicas de inclusión y valoración de las vivencias que se muestran en el proceso creativo.

La reflexión y diálogo entre distintas culturas permite conocer desde un contexto latinoamericano lo arraigadas que están las prácticas sociales hegemónicas, que mantiene y reproduce la violencia de género. Sin embargo, el plantear elementos didácticos con enfoque de género permitió visibilizar y reconocer la importancia de los vínculos y los afectos como una forma de desarrollo de subjetividades.

\section{Conclusiones: Estudio escuela primaria en Santiago de Chile}

El proceso de reflexión artística presentado en las dos experiencias de este artículo propone un proceso que va más allá de la asignatura de Artes Visuales y traspasa el espacio áulico para abordar problemáticas sociales, que afectan no solo al estudiantado, sino a todas las personas que conforman la comunidad escolar. Se hace vital proponer aulas desjerarquizadas, pensar al profesorado en sintonía con sus estudiantes y proponer aulas de trabajo colectivo y respetuosas con las subjetividades.

A partir del caso 1 se establece una resignificación de la vida cotidiana a partir de la música y se proponen nuevas formas de abordaje de las problemáticas sociales cotidianas, con un ejercicio creativo colectivo, que empuja al estudiantado a llevar a cabo procesos reflexivo-críticos.

Esto podría dar espacio a concebir "Una institución flexible en tiempos, secuencias, metodologías, modelos de evaluación, sistemas de convivencia, etc., que toma en cuenta la diversidad de la condición adolescente y juvenil (de género, cultura, social, étnica, religiosa, territorial,etc.)" (Tenti, 2000, p. 9). Esto propicia avanzar en la lectura de las experiencias expuestas, como actividades que develan la necesidad de incorporar elementos reflexivos profundos en el progreso y enseñanza de las Artes Visuales que permita el desarrollo de 
posicionamientos críticos frente a situaciones de violencia de género, configuración de identidad, subjetividades colectivas y crítica a los estereotipos de género, pero considerando las propias experiencias del estudiantado que permita cargar de sentido a las experiencias escolares. Esto permite desarrollar una reflexividad posicional, la que involucra el propio mundo de las personas, tanto de las que investigan como de quienes colaboran con las investigaciones.

Las reflexiones y diálogos que emergieron a partir de las experiencias que conforman este artículo han permitido iniciar un proceso de búsqueda de posicionamientos críticos frente a hechos como el Estallido Social y el Mayo Feminista en Chile, como coyunturas que generan debate social y permean a los espacios escolares. Ejemplo de esto es que, en el acto de término del ciclo primario en la escuela, uno de los estudiantes subió con una bandera negra en sus brazos, en reconocimiento de las personas mutiladas en el estallido social. Para complementar lo anterior quisiéramos compartir unas ideas que reflejan el desarrollo del 6to año básico del 2018 y culmina con las siguientes palabras el 2019, dirigidas a la docente de Artes Visuales que formó parte del equipo de investigación de las experiencias expuestas en el artículo:

Agradezco lo que hizo por nosotros, por apoyarnos y por sobretodo hacerme sentir respetada y queriendo provocar un cambio en nosotros. Y le aseguro que me acordaré de usted hasta que Chile logre hacer un cambio. (Estudiante del curso participante de la investigación)

Si bien hay una preocupación en el contexto áulico de Artes Visuales frente a temáticas de género no es una generalidad en la escuela, lo que se presenta como una dificultad dentro del estudio porque limita no solo las posibilidades de trabajar transversalmente la perspectiva de género (García, Díaz y Hernández, 2020). De esta manera una evidente limitación es que las experiencias mostradas en el artículo quedan en el anecdotario o como una experiencia aislada dentro de la comunidad educativa.

Sin embargo, es posible realizar recomendaciones que se pueden establecer a partir del artículo y el proceso de investigación. Se propone la conformación de equipos de trabajo interdisciplinario dentro de la escuela para abordar temáticas de género vinculadas a las disciplinas de estudio, llevar a cabo acciones y evaluar constantemente las iniciativas (Subirats, 2017). Lo anterior se puede articular como instancias reflexivas a partir de los procesos creativos. También se abren interrogantes para futuros estudios en relación con ¿Cuáles son las posibilidades y potencialidades de las experiencias artísticas con temáticas 
de género en otras asignaturas de la escuela? ¿Es posible incorporar estas experiencias en áreas de acompañamiento socioemocional en las escuelas? ¿Es posible, desde estas experiencias artístico-colaborativas, instaurar de manera transversal el enfoque de género en las escuelas? A partir de estas preguntas y del desarrollo de este artículo, se intentará seguir avanzando hacia la conformación de aulas más seguras, inclusivas y que desarrollen un proceso creativo y reflexivo en torno a las temáticas de preocupación social, que den espacio a procesos narrativos más allá de la oralidad o de la escritura.

\section{Referencias}

Barbosa, Ana. (2002). Arte, educación y reconstrucción social, Cuadernos de Pedagogía (312), 56-58.

Belausteguigoitia, Marisa., y Mingo, Aracely. (1999). Fuga a dos voces. Ritmos, contrapuntos y superposiciones del campo de los estudios de género y la educación. En Marisa Belausteguigoitia y Aracely Mingo (Comp.), Géneros Prófugos (pp.13-53). México: UNAM.

Bejarano, María., Martínez, Irene., y Blanco, Montserrat. (2019). Coeducar hoy. Reflexiones desde las pedagogías feministas para la despatriarcalización del currículum. Tendencias Pedagógicas, 34, 37-50. Recuperado de https://revistas.uam.es/tendenciaspedagogicas/article/view/tp2019.34.004

Blanco, Paloma. (2001). Explorando el terreno. En Paloma Blanco (Eds.), Modos de hacer. Arte crítico, esfera pública y acción directa (pp. 5-40). Salamanca: Ediciones Universidad de Salamanca.

Birchall, Michael. (2017). Situating Participatory Art Between Process and Practice. The Art of Taking Part. Arkin Bulletin, 7, 56-74. Recuperado de https://www.arken.dk/wpcontent/uploads/2017/04/situating-participatory-art-between..-by-michaelbichall bulletin-2017.pdf

Bourdieu, Pierre. (2000). Sobre el poder simbólico. en Intelectuales, política y poder, (traducción de Gutiérrez, A.). Buenos Aires: UBA/ Eudeba.

Cabello, Cristeva. (2018). Educación no sexista y binarismo de género. Agitaciones feministas y disidencias sexuales secundarias en la escuela. En Faride Zerán (Ed.), Mayo Feminista. La rebelión contra el patriarcado (pp.21-34). Santiago: LOM.

De Lauretis, Teresa. (1984). Alice Doesn’t. Bloomington: Indiana University Press.

Diaz-Romero, Pamela. (2020). Del 8M al 18/10: continuidades, cambios y nuevas oportunidades. En La demanda ciudadana por una nueva democracia (pp. 187-198). Santiago: Sur ediciones. 
Eisner, Elliot. (2017). El arte y la creación de la mente. El papel de las artes visuales en la transformación de la conciencia. Barcelona: Paidós.

Faure, Edgar., Herrera, Felipe., Kaddoura, Abdul-Razzak., Lopes, Henri., Petrovski, Arthur., Rahnema, Majid., y Ward, Frederick. (1973). Aprender a ser. La educación del futuro. Madrid: Alianza/UNESCO.

Flores-Kastanis, Eduardo., Montoya-Vargas, Juny., y Suárez, Daniel H. (2009). Investigaciónacción participativa en la educación latinoamericana: un mapa de otra parte del mundo. Revista mexicana de investigación educativa, 14(40), 289-308. Recuperado de http://www.scielo.org.mx/scielo.php?script=sci arttext\&pid=S1405$\underline{66662009000100013}$

García, Jorge., Díaz, Dalia., y Hernández Claudia. (2020). La transversalización de la perspectiva de género como una herramienta de intervención en educación. Revista Investigium IRE: Ciencias Sociales y Humanas, 11(1), pp.69-82. Recuperado de https://dialnet.unirioja.es/servlet/articulo?codigo $=7836465$

García-Huidobro, Rosario., y Montenegro, Catalina. (2020). Las prácticas artísticas con enfoques feministas como experiencias educativas que promueven la transformación social. Revista Electrónica Educare, 24(1), 1-16. Recuperado de https://www.scielo.sa.cr/scielo.php?pid=S1409-42582020000100440\&script=sci arttext

Gerber, Elisabet. (2020). Las Tesis y la revolución de las hijas. En La demanda ciudadana por una nueva democracia (pp. 199-201). Santiago: Sur ediciones.

Huerta, Ricard. (2016). Transeducar. Arte, docencia y derechos LGTB. Madrid: Egales.

Lamas, Marta. (1986). La antropología feminista y la categoría "género", Nueva Antropología, 8(30), 173-198. Recuperado de https://www.redalyc.org/pdf/159/15903009.pdf

Ledezma, Miguel., Reyes, Eric., y Caporal, Julia. (2017). Las relaciones entre método de investigación-acción participativa y el arte de interacción social. Alcances y riesgos. Tercio Creciente, (13), 97-114. Recuperado de https://revistaselectronicas.ujaen.es/index.php/RTC/article/view/3418

Lewin, Kurt. (1946). La investigación acción participativa. La investigación-acción y los problemas de las minorías. Disponible en https://dialnet.unirioja.es/descarga/articulo/2903452.pdf

Liviana, Aïcha. (2020). Feminismo y revolución, crónica de una inquietud, Santiago 2019. Fragmentos de una paz insólita. Santiago: Metales Pesados.

Macbeth, Douglas. (2001). On "reflexivity" in qualitative research: Two readings, and a third. Qualitative inquirí, 7(1), 35-68.

Maceira, Luz. (2008). El Sueño y la Práctica de Sí: Pedagogía Feminista: Una Propuesta. México D.F: El Colegio de México, A .C., Biblioteca Miguel Cosio Villegas. 
Marí, Rosa., Barranco, Rut., y Martínez, Irene. (2019). Educar en sexualidades e igualdad en educación social desde una perspectiva feminista. María En Bejarano y Rosa Marí (Dir.), Educación en sexualidad e igualdad. Discursos y estrategias para la formación de docente y educadores sociales (pp. 31-50). Castilla: Dikinson.

Ministerio de las Culturas, las Artes y el Patrimonio (Mincap). (2020). Perspectiva de género en Programa Acciona: Una propuesta desde la región de Los Lagos. Santiago: Ministerio de las culturas, las artes y el Patrimonio- Colección Educación Artística. Santiago: Ministerio de las Culturas, las Artes y el Patrimonio.

Ministerio de Educación. (2013). Bases curriculares Artes Visuales. Chile: MINEDUC.

Mohanty, Chandra. (2008). De vuelta a "Bajo los ojos de Occidente": la solidaridad feminista a través de las luchas anticapitalistas. En Liliana Suarez y Rosalva Hernández (Eds.), Descolonizando el feminismo: Teorías y prácticas desde los márgenes (pp. 407-464). Barcelona: Cátedra.

Montenegro, Catalina., y Corvalán, Alejandra. (2020). Desplazamientos desde las pedagogías feministas: reflexiones posibles para un ejercicio docente situado. FEMERIS: Revista Multidisciplinar de Estudios de Género, 5(3), 8-29. Recuperado de https://erevistas.uc3m.es/index.php/FEMERIS/article/view/5760

Montenegro, Catalina. (2019a). Derivas feministas para una educación contemporánea. En Gabriel Hoecker y Magdalena Dardel (Eds.), Chile despertó. Cuaderno pedagógico (pp. 20-24). Santiago.

Montenegro, Catalina. (2019b). La creación artística como proceso reflexivo del SER en femenino: el caso de la escuela chilena. Revista estudios sobre arte actual, (7), 113-118.

Montenegro, Catalina. (2019c). Cartografías colectivas como espacios de reflexión visual: territorios, experiencias y contextos. Acta IV Congreso Internacional de Investigación en Artes Visuales ANIAV 2019, IMAGEN [N] VISIBLE], 517-523. Recuperado de https://riunet.upv.es/handle/10251/129793

Montenegro, Catalina. (2018a). Propuesta de didáctica feminista para la enseñanza escolar de las artes visuales: pensar el espacio escolar de otra manera. Revista Estudios pedagógicos, 44(3), 289-302. Recuperado de https://scielo.conicyt.cl/scielo.php?script=sci arttext\&pid=S0718-07052018000300289

Montenegro, Catalina. (2018b). Cultura visual y pedagogías feministas: repensando los espacios educativos. En Estibaliz Aberasturi-Apraiz, Amaia Arriaga e Idoia MarcellánBaraze, (Coords.), Arte, lustración y Cultura Visual. Diálogos en torno a la mediación educativa crítica dentro y fuera de la escuela (pp. 347-353). Universidad Pública de Navarra, Pamplona.

Montenegro, Catalina. (2015). Del saber de mujeres machi al saber docente: una investigación biográfico narrativa (Tesis para optar al grado de Doctor en Arte y Educación). Universidad de Barcelona, Barcelona, España. 
Moreno, Ascensión. (2016). La mediación artística. Artes para la transformación social, la inclusión social y el desarrollo comunitario. Barcelona: Octaedro.

Munévar, Dora. (2015). Lecturas normativo-experienciales y prácticas pedagógicas plurales sobre inclusión académica. Mundos plurales, Revista Latinoamericana de Políticas y Acción Pública, 2(1), 55-76. Recuperado de https://revistas.flacsoandes.edu.ec/mundosplurales/article/view/1911

Muñoz, Ana y Lira, Andrea. (2020). Política educacional desde el feminismo: conversaciones iniciales. Núcleo milenio, experiencias de los estudiantes de la educación superior. Recuperado de http://nmedsup.cl/wp-content/uploads/2020/08/policy-brief-n5.pdf

Navarro, Luis. (2020). Crisis social y pactos en educación. En La demanda ciudadana por una nueva democracia (pp.165-186). Santiago: Sur ediciones.

Orner, Mimi. (1999). Interrumpiendo los llamados para una voz de el y la estudiante en la "educación liberadora". Una perspectiva posestructuralista feminista. En Marisa Belausteguigoitia y Aracely Mingo (Comp.), Géneros Prófugos (pp.117-134). México: UNAM.

Palacios, Alfredo. (2009). El arte comunitario: origen y evolución de las prácticas artísticas colaborativas. Papeles de arteterapia y educación artística para la inclusión social, 4, 197-211. Recuperado https://revistas.ucm.es/index.php/ARTE/article/view/ARTE0909110197A

Ramos, David (Ed.). (2019). Miradas caleidoscópicas: educación artística visual en las culturas contemporáneas. Bogotá: Universidad Pedagógica Nacional.

Reyes-Housholder, Catherine., y Roque, Beatriz. (2019). Chile 2018: Desafíos al poder de género desde la calle hasta la Moneda. Revista de Ciencia Política, 39(2), 191-215.

Richard, Nelly. (2018). La insurgencia feminista de mayo 2018. En Faride Zerán (Ed.), Mayo Feminista. La rebelión contra el patriarcado (pp.115-126). Santiago: LOM.

Rodríguez, Carlos. (2015). Transigrafías. Caminar como práctica artístico-pedagógica. Revista de Estudios Urbanos y Ciencias Sociales, 5(1), 35-56. Recuperado de https://dialnet.unirioja.es/servlet/articulo?codigo=5081019

Rodrigo, Javier., y Collados, Antonio. (2015). Retos y complejidades de las prácticas artística colaborativas y las pedagogías colectivas. Pulso, (38), 57-72. Recuperado de https://revistas.cardenalcisneros.es/index.php/PULSO/article/view/187

Sánchez, Mercedes., Penna, Melani., y De la Rosa, Belén. (2016). Somos como somos. Deconstruyendo y transformando la escuela. Madrid: Catarata.

Seoane, Viviana., y Severino, Moira. (2019). Género, sexualidades y educación. Intersecciones necesarias para una educación emancipadora. Descentrada, 3(1), 1-6. Recuperado de https://dialnet.unirioja.es/servlet/articulo?codigo=6879340 
Subirats, Marina. (2017). Coeducación, apuesta por la libertad. Barcelona: Octaedro.

Subirats, Marina., y Tomé, Amparo. (2013). Balones fuera. Reconstruir los espacios desde la coeducación. Barcelona: Octaedro.

Tenti, Emilio. (2000). Culturas juveniles y cultura escolar. Documento presentado al Seminario Escola Jovem: un novo olhar sobre o ensino médio, organizado por el Ministerio da Educaçao. Secretaria de Educaçao Média e Tecnológica. Coordenaçao-Geral de Ensino Médio (Brasilia, junio 7-9 de 2000).

Tomé, Amparo y Rambla, Xavier. (2001). Contra el sexismo: coeducación y democracia en la escuela. Barcelona: Síntesis.

Troncoso, Lelia., Follegati, Luna., y Stutzin, Valentina. (2019). Más allá de una educación no sexista: aportes de pedagogías feministas interseccionales. Pensamiento Educativo, 56(1), 1-15. Recuperado de http://rchd.uc.cl/index.php/pel/article/view/23411

Walsh, Catherine. (2014). Introducción, Lo pedagógico y decolonial: Entretejiendo caminos. En Catherine Walsh (ed.), Pedagogías decoloniales. Prácticas insurgentes de resistir, (re)existir y (re)vivir (pp.23-69). Querétaro: En cortito que's pa'largo

Zerán, Faride. (2018). Prólogo Escrituras rebeldes para tiempos de cambio. En Zerán, Faride. (Ed.), La rebelión contra el patriarcado (pp. 9-20). Santiago: LOM. 
Revista indizada en

sciplo redalyc latindex DOA J

Distribuida en las bases de datos:

- Dialnet

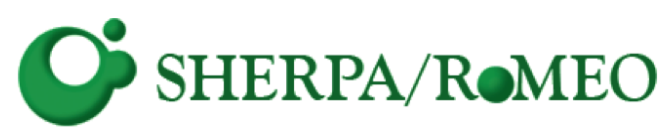

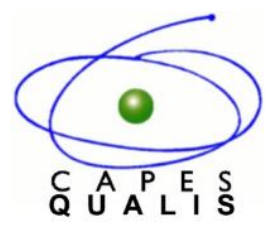

MIAR 